ARTICLE

\title{
Photoacoustics of single laser-trapped nanodroplets for the direct observation of nanofocusing in aerosol photokinetics
}

\author{
Johannes W. Cremer ${ }^{1}$, Klemens M. Thaler ${ }^{2}$, Christoph Haisch ${ }^{2} \&$ Ruth Signorell ${ }^{1}$
}

Photochemistry taking place in atmospheric aerosol droplets has a significant impact on the Earth's climate. Nanofocusing of electromagnetic radiation inside aerosols plays a crucial role in their absorption behaviour, since the radiation flux inside the droplet strongly affects the activation rate of photochemically active species. However, size-dependent nanofocusing effects in the photokinetics of small aerosols have escaped direct observation due to the inability to measure absorption signatures from single droplets. Here we show that photoacoustic measurements on optically trapped single nanodroplets provide a direct, broadly applicable method to measure absorption with attolitre sensitivity. We demonstrate for a model aerosol that the photolysis is accelerated by an order of magnitude in the sub-micron to micron size range, compared with larger droplets. The versatility of our technique promises broad applicability to absorption studies of aerosol particles, such as atmospheric aerosols where quantitative photokinetic data are critical for climate predictions.

\footnotetext{
${ }^{1}$ Department of Chemistry and Applied Biosciences, Laboratory of Physical Chemistry, ETH Zurich, Vladimir-Prelog-Weg 2, CH-8093 Zurich, Switzerland. ${ }^{2}$ Laboratory for Applied Laser Spectroscopy, Chair of Analytical Chemistry, Technical University of Munich, Marchioninistrasse 17, D-81377 Munich, Germany. Correspondence and requests for materials should be addressed to R.S. (email: rsignorell@ethz.ch).
} 
U nderstanding fundamental processes that govern the reaction dynamics of gas phase, aerosol and cloud processes is crucial for the advancement of global atmospheric chemistry modelling ${ }^{1-14}$. Much of the chemistry occurring in the Earth's atmosphere is driven by sunlight. Photochemical reactions, in which aerosol particles or droplets act as the active reaction medium, can be highly complex because they are influenced by optical phenomena, transport properties and surface effects ${ }^{2}$. Optical phenomena play a fundamental role in light-initiated particle processes since the radiation flux within the particles determines the activation rate of the photochemically active species.

Focusing of electromagnetic radiation inside small particles leads to an enhancement of the overall light intensity, compared with the intensity of the incident radiation and to structuring and localization of the internal optical fields ${ }^{15-23}$. These phenomena depend strongly on the particle size, the particle composition and the wavelength of electromagnetic radiation. The fundamental influence of the enhanced electromagnetic energy density on the rate of photochemical reactions in micro- and nanodroplets has been recognized and calculations have provided limited evidence for enhanced photochemical rates ${ }^{24-27}$. Experimental results remain inconclusive concerning the influence of light enhancement on the kinetics, mainly because direct observation of the actual photoactive step was not possible $23,28-34$. The observation of size-dependent effects in ensembles of aerosol or emulsion droplets is often hindered because the droplet size distribution cannot be varied and determined with the necessary accuracy. However, even single-droplet techniques have so far not provided size-dependent photolysis rates because the direct measurement of the population decay of the photoactive substance was not possible.

Elastic light scattering is sensitive enough to allow measurements on single sub-micron-sized droplets, but the information content is not specific enough to extract size-dependent rates. Raman spectroscopy, by contrast, could provide specific information but it comes with the disadvantage of low sensitivity (long averaging times), which would make its application to study processes in single submicrometre droplets where nanofocusing becomes important very challenging. Single-droplet fluorescence studies require a fluorescing compound, which strongly restricts its applicability. Furthermore, the fluorescence depletion is not always a reliable measure of the population decay of the photoactive species because of varying quenching efficiencies. The recently presented cavity ring-down studies on single droplets provide information on the extinction but not directly on the droplet absorption ${ }^{35,36}$. Even in combination with light scattering measurements, the determination of rates in nanodroplets is likely prohibited by the uncertainty of the derived absorption.

This study reports the direct observation of light nanofocusing on the photokinetics in nanometre- to micron-sized droplets in the ultraviolet/visible (UV/VIS) range. To this end, we introduce single-droplet photoacoustic (PA) absorption spectroscopy, allowing the direct detection of the population decay of the photoactive substance. PA spectroscopy has been successfully used for the investigation of ensembles of aerosol particles ${ }^{37-40}$, but its applicability to single aerosol particle studies has been controversial and has not previously been realised experimentally. Here we demonstrate the feasibility of single-droplet PA spectroscopy in combination with laser trapping, and provide direct experimental evidence for the size-dependence of the photolysis rate in model aerosol droplets due to nanofocusing effects. The results are compared with simulations using classical cavity electrodynamics.

\section{Results}

Principle of single-droplet PAs. The two experimental set-ups, using a microphone and a quartz tuning fork, respectively, for resonant single-droplet PA measurements, are sketched in Fig. 1a,b (see Methods). For the droplet absorption experiments, we use a $\lambda=445 \mathrm{~nm}$ excitation laser (Nichia laser diode NDB7112E) of variable power $(0.3-40 \mathrm{~mW})$ modulated at the resonance frequency of the PA-resonator and the tuning fork, respectively. The resonance frequency and the $Q$-factor of the PA-resonator and the tuning fork are $3.97 \mathrm{kHz}$ and $\sim 8.9$, and $32.7 \mathrm{kHz}$ and $\sim 8,000$, respectively (Supplementary Fig. 1). The power of the excitation laser is recorded by a power meter after passing the PA cell and the tuning fork, respectively (Supplementary Fig. 2). The amplified PA signals are averaged over either $30 \mathrm{~ms}$ or $200 \mathrm{~ms}$. For single-particle trapping, we use a counter-propagating optical tweezer built from a continuous laser beam of $\lambda=660 \mathrm{~nm}$ of $\sim 200 \mathrm{~mW}$ (Laser Quantum, Opus 660) (Supplementary Fig. 2). Such multiple beam optical traps allow trapping of sub-micron droplets, and combine the advantage of a comparatively simple set-up with high trapping stability and tight particle confinement $(<100 \mathrm{~nm})^{41-43}$. Droplets are trapped by gradient forces pointing towards the trap centre for all translational degrees of freedom. Single droplets are captured in the trap centre from a plume of aerosol generated by a nebulizer (see Methods). The droplet size is determined from laser light elastically scattered by the droplet ${ }^{41,44}$ (see Methods, Supplementary Fig. 3). Figure 1e shows an example for light scattering measurements for droplet sizing. In the microphone set-up (Fig. 1a), the trap centre is located in the middle of the PA-resonator above the microphone ${ }^{45}$. The trapping and excitation lasers enter and exit the cell through wideband, anti-reflective windows coated for the respective wavelengths. The CMOS camera for particle imaging and light scattering measurements is placed perpendicular to the excitation and trapping laser. The aerosol inlet and outlet are on the side of the PA cell outside the resonator.

In the tuning fork set-up (Fig. 1b), the droplet is trapped between the tines of the fork with collinearly aligned excitation laser and trapping laser beams. The CMOS camera for particle imaging and light scattering measurements is placed opposite the tines of the fork. Figure 1c,d shows images of a single droplet trapped in between the tines and of a droplet ensemble flowing through the tines, respectively. The principal attractiveness of the tuning fork derives from its high detection sensitivity (very high $Q$-factors) and low sensitivity to environmental acoustic noise ${ }^{46}$. In our set-up, we mainly profit from the ease of combining it with laser trapping and light scattering measurements, as well as from the fact that it is chemically inert.

PA response of a single droplet. Figure 2 provides typical noise levels, background signals and a proof for single-droplet detection. Figure 2a illustrates the noise level and the background signal for the empty trap with the trapping laser on. With the excitation laser off $(-5 s<t<0 s)$, the background signal (average) and noise level (1 s.d.) are $\sim 2.2 \pm 1.2 \mu \mathrm{V}$. Once the excitation laser is turned on at time $t=0 \mathrm{~s}$, a background signal of $B S=5.3 \mu \mathrm{V}$ with a noise level of $N L=1.7 \mu \mathrm{V}$ is recorded. The background signal is caused by excitation laser light scattered from the cell walls and hitting the microphone. Blocking the trapping laser (that is, disabling the trap) leaves the noise, as well as the background unchanged.

Figure $2 \mathrm{~b}$ shows the same as Fig. 2a but with a single VIS441/tetraethylene glycol (TEG) solution droplet in the trap. The PA signal reaches a maximum $\left(S_{\max }\right)$ just after the excitation laser is turned on and then decreases exponentially as the VIS441 absorber undergoes photolysis. In Fig. 2b, the trap is disabled at 
a

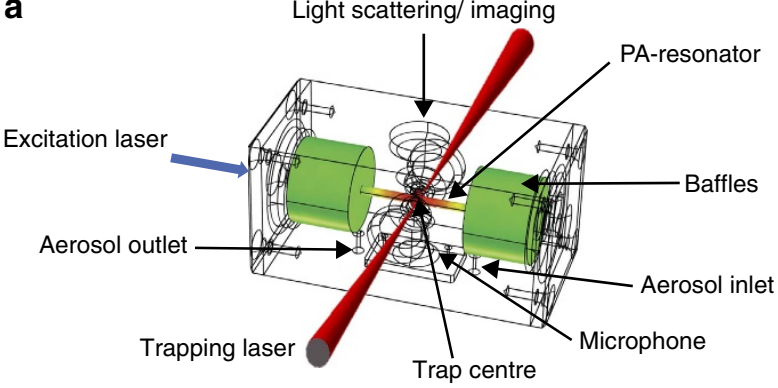

b
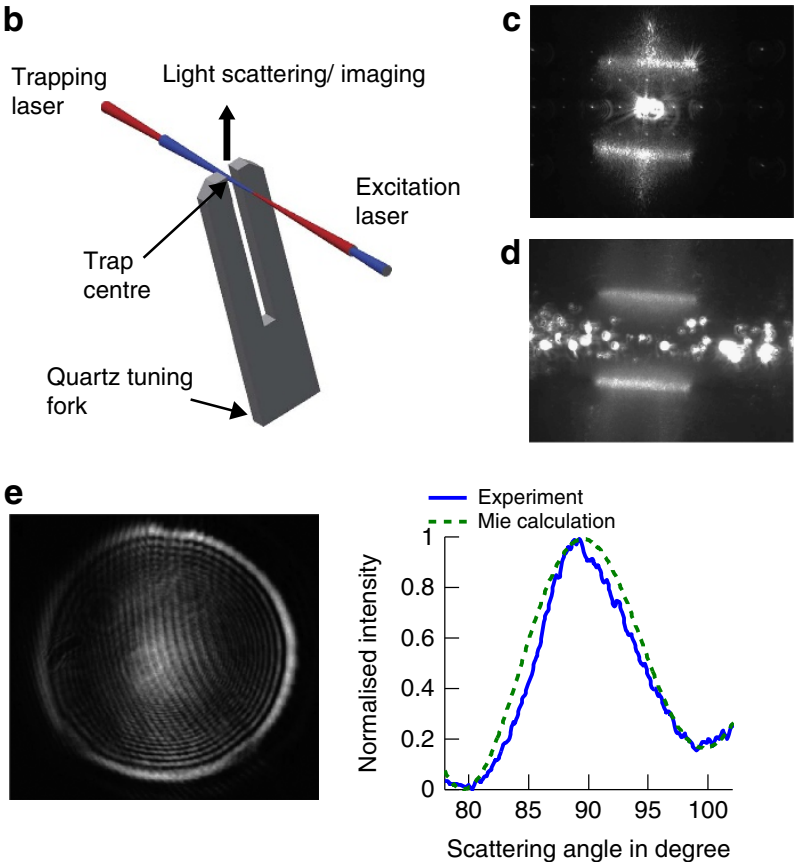

Figure 1 | Sketch of the experimental single-droplet PA set-ups.

(a) Microphone set-up with PA-resonator, excitation laser, trapping laser and light scattering measurements. The colours in the PA cell indicate that the acoustic mode has its maximum amplitude (red) in the vicinity of the microphone and a value close to zero (green) in the region of the acoustical baffles. (b) Tuning fork set-up. (c) Snapshot of a single droplet trapped between the tines of the tuning fork (view from top). Note that the droplet $(\sim 1 \mu \mathrm{m})$ is much smaller than the detection volume between the tines $(\sim 0.3 \times 0.34 \times 2 \mathrm{~mm})$. (d) Snapshot of an ensemble of droplets flowing in between the tines from left to right. (e) Light scattering image as recorded by the CMOS camera (left) with experimental and calculated phase function (right) for a droplet with a radius of $a=530 \mathrm{~nm}$.

$t=7 \mathrm{~s}$ which leads to the immediate loss of the droplet and to a decrease of the PA signal to the background signal BS. This proves that the signal between $0 \mathrm{~s}<t<7 \mathrm{~s}$ indeed comes from a single droplet. The signal to noise ratio $\frac{S}{N L}=\frac{S_{\max }-\mathrm{BS}}{N L}$ depends on the power $P$ of the excitation laser, the concentration of the solution and the droplet size. Figure 3 shows exemplary experimental data for solution droplets of different size excited with different laser powers $P$. With the tuning fork set-up, we find an improvement in the $\frac{S}{N L}$ ratio of a factor of $\sim 3$ compared with the microphone set-up. Note that the PA signal is caused by absorptive heating of the droplet and subsequent heat transfer to the surroundings. Evaporation of the solvent does not occur.

Detection limit. The minimum absorbance detectable with the single-particle PA set-ups at a given power of the excitation laser
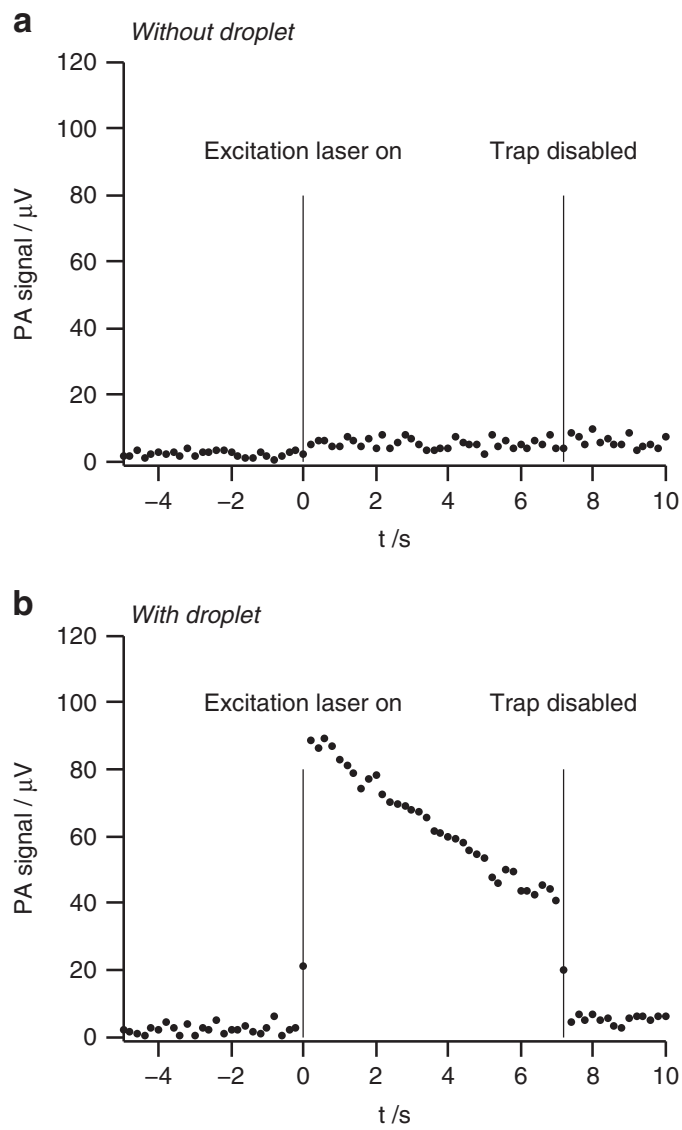

Figure 2 | Typical noise levels and background signals for the microphone set-up. (a) For the empty trap, identical noise levels and background signals are recorded for a pure TEG solvent droplet in the trap (data not shown). (b) With a VIS441/TEG solution droplet in the trap. At $t=0 \mathrm{~s}$, the excitation laser $(445 \mathrm{~nm})$ is switched on and at $t=7 \mathrm{~s}$ the trapping laser is switched off.

can be estimated from the PA signal and the single-droplet absorbance assuming that the noise level $N L$ is the detection limit (see Methods, Calculation of droplet absorbance). As an example, we use the PA signal at $t=0\left(S_{\max }\right)$ of the $530 \mathrm{~nm}$ droplet shown in Fig. 3a recorded at a laser power of $2.8 \mathrm{~mW}$ with an averaging time of $200 \mathrm{~ms}$. The refractive index of this droplet, $n+i k=1.463+i \cdot 0.0062$ (Methods and Supplementary Figs 4 and 5), yields an absorption cross-section of $C_{\mathrm{abs}}=0.22 \mu \mathrm{m}^{2}$. $\left(S_{\max }-B S\right)=33.3 \mu \mathrm{V}$ corresponds to an equivalent absorbance of $A=1.8 \times 10^{-5}$. From the measured $N L$ of the $530 \mathrm{~nm}$ droplet of $N L=1.7 \mu \mathrm{V}$, we derive a minimum absorbance $A_{\min }=9 \times 10^{-7}$ detectable with the microphone set-up. The improvement in $\frac{S}{N L}$ by a factor of $\sim 3$ for the tuning fork set-up reduces the detection limit to $A_{\min } \sim 3 \times 10^{-7}$, a minimum detectable absorption coefficient of $\alpha_{\min }=0.0074 \times 10^{-6} \mathrm{~m}^{-1}$ or a minimum detectable absorption cross-section $C_{\text {abs,min }}=0.0037 \mu \mathrm{m}^{2}$ (laser power of $2.8 \mathrm{~mW}$ and averaging time of $200 \mathrm{~ms}$ ) (see Methods equations (2)-(4)). The equivalent particle radius of $146 \mathrm{~nm}$ corresponds to a probe volume of $13 \mathrm{al}$. This far exceeds the performance of typical spectrometers $\left(A_{\min } \sim 10^{-3}-10^{-4}\right)$, and is at least comparable to the most sensitive laser spectroscopic absorption measurements for macroscopic probe volumes. Note that in our set-up, this sensitivity is achieved for small (attolitre) probe volumes and very short $(<<1 \mathrm{~s})$ measurement times. Both can be further improved by increasing the laser power. 
a

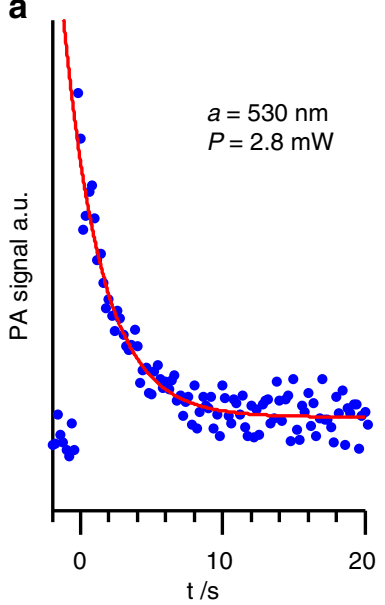

C

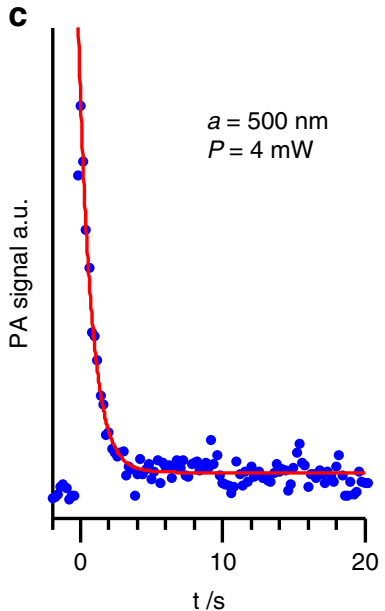

b

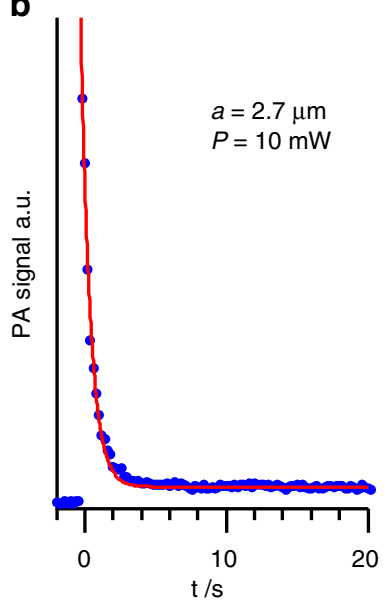

d

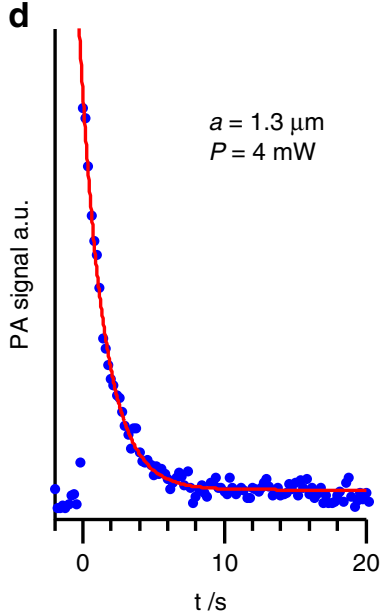

Figure 3 | Exemplary PA signals of VIS441/TEG solution droplets as a function of time. The decay of the signal is caused by photolysis of the solute. The experimental data (blue dots) are recorded for different droplet radii $a$ and different power $P$ of the excitation laser. $(\mathbf{a}, \mathbf{b})$ Recorded with the microphone set-up. (c,d) Recorded with the tuning fork setup. The red lines are fits to the experimental data providing experimental first half-lives $t_{1 / 2}$ (see Fig. 4).

Size-dependent photokinetics. The photokinetics in small droplets do not follow simple pseudo first order kinetics because the light intensity distribution inside the droplets is time dependent; that is, because of the concentration dependence of the nanofocusing. Therefore, we use the same initial concentration for all experiments so that the first half-life can be used as a measure for the size-dependence of the photokinetics. With our PA set-up, we directly measure the decay in absorption resulting from the population decay of the photoactive substance. Diffusion is so fast in the droplets $\left(\sim 10 \mu \mathrm{m}^{2} \mathrm{~s}^{-1}\right)$ that concentration gradients cannot build up and homogeneous concentrations for the solute can be assumed at all times. A model for the droplet photokinetics under these conditions is provided in Methods (Calculation of droplet photokinetics).

The model prediction (full blue line in Fig. 4a) shows a strong droplet size-dependence with a maximum in the inverse first halflife $\frac{1}{t_{1 / 2}}$ at a droplet radius of $\sim 0.5 \mu \mathrm{m}$. Pronounced increases in $\frac{1}{t_{1 / 2}}$ and fluctuations due to resonances are observed over the droplet size range from $\sim 50 \mathrm{~nm}$ to $\sim 1.2 \mu \mathrm{m}$. In this size range, the increase of the rate is caused by the enhancement
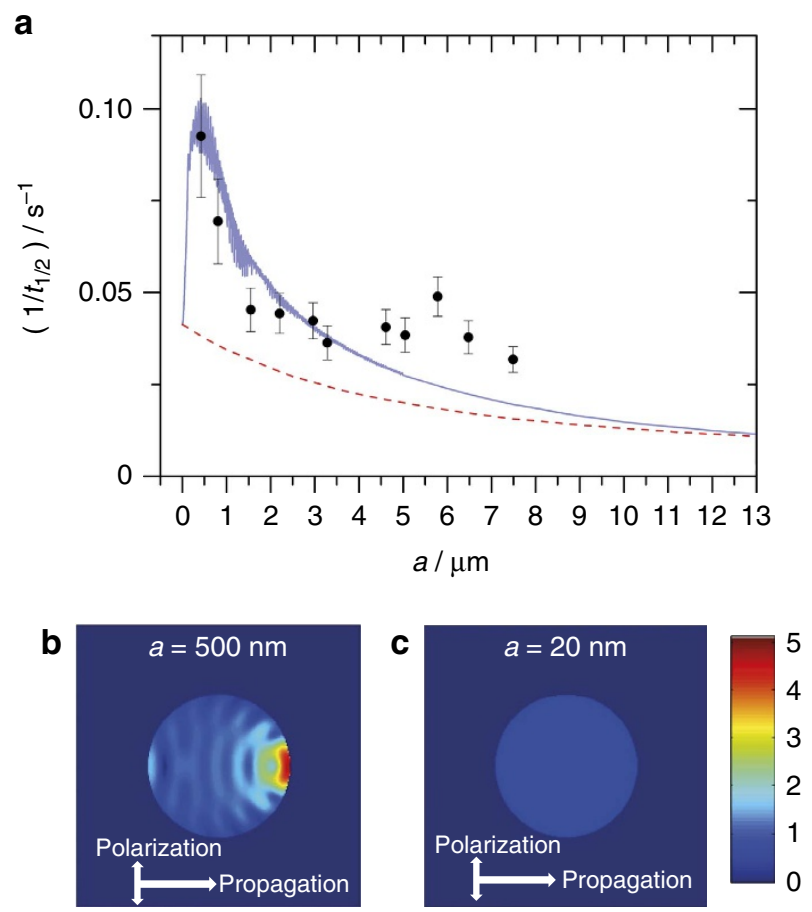

Figure 4 | Size-dependent photokinetics. (a) Inverse first half-lives $\frac{1}{t_{1 / 2}}$ as a function of the droplet radius for a laser power of $1 \mathrm{~mW}$. Black circles: statistically evaluated experimental data. Error bars show 1 s.d. Full blue line: model prediction including nanofocusing and scattering effects. The calculations are for a quantum yield of $7 \times 10^{-6}$. Dashed red line: model prediction for a hypothetical bulk limit, that is, excluding contributions from nanofocusing and scattering. Distribution of the light intensity inside droplets at $t=0 \mathrm{~s}$ for (b) a $0.5 \mu \mathrm{m}$ droplet and (c) a $20 \mathrm{~nm}$ droplet. The colour scheme is relative to an incident light intensity of 1 .

of the internal electromagnetic field intensity through focusing of the light inside the droplet. Figure $4 \mathrm{~b}$ shows the distribution of the light intensity inside a $\sim 0.5 \mu \mathrm{m}$ droplet at $t=0 \mathrm{~s}$. The enhancement of the overall intensity and the local variation of the intensity are pronounced. The inverse half-life at a droplet radius of $\sim 0.5 \mu \mathrm{m}$ is increased by a factor of $\sim 2.5$ compared with the infinitely small droplet limit. The kinetics in these small droplets is no longer influenced by nanofocusing inside the droplet or light scattering by the droplet as visualized by the internal field intensity in Fig. 4c. The inverse half-life of larger droplets $(>6 \mu \mathrm{m})$ exhibits only a weak size-dependence but decreases continuously (towards zero for infinitely large particles). The rate for these large droplets is determined by the balance between the decay of the absorber and the rise of the decay rate with time. As the photolysis proceeds, the penetration depth of the light and hence the internal field intensity increases. As in the case of very small droplets, nanofocusing is not important for very large droplets. Large droplets essentially represent the behaviour of thin bulk films with the same effective thickness as the droplet. The $\frac{1}{t_{1 / 2}}$ increases by a factor of $\sim 10$ for a $0.5 \mu \mathrm{m}$ compared with a $13 \mu \mathrm{m}$ droplet, which implies a substantial increase in the rate of sub-micron-size droplets relative to bulk. The dashed red line in Fig. 4a simulates the behaviour of a hypothetical droplet excluding the influence of nanofocusing and light scattering but still accounting for the droplet-size-dependent absorption (see Methods, Calculation of droplet photokinetics). This curve represents bulk behaviour. The comparison with the full blue line 
clearly shows the pronounced influence of light focusing on the rate in the sub-micron to micron size range.

The statistically evaluated experimental first half-lives (black circles in Fig. 4a) are determined from time-dependent PA measurements (see Methods, Statistical analysis). The experimental results clearly follow the size-trend predicted by the model (full blue line). The pronounced maximum of $\frac{1}{t_{1 / 2}}$ at a droplet size of $\sim 0.5 \mu \mathrm{m}$ is clearly visible even though the data scatter notably below $\sim 1 \mu \mathrm{m}$ (Supplementary Fig. 6) mainly because of the uncertainty in the droplet size determination (Methods, Droplet Sizing). Our experimental data show somewhat higher values of the inverse half-life for larger droplets than the model prediction. Deviations from the model assumptions including modified PA response in large droplets ${ }^{47}$ could potentially account for this. We have recently introduced a broad-band scattering method for accurate sizing of submicrometre particles, which will allow us in future to significantly reduce the size uncertainty in the submicrometre range (unpublished data). However, already at the current level of accuracy, the data in Fig. 4 a provide the first direct observation of the strong influence of nanofocusing of light on the photokinetics in droplets.

\section{Discussion}

The experimental results in Fig. 4 confirm a strong size-dependence of the rate of photochemical reactions in droplets. This optical phenomenon shows the most pronounced effect in the submicrometre to micrometre droplet size range for electromagnetic radiation in the UV to VIS range, that is, for the relevant frequency range in atmospheric processes. Classical cavity electrodynamics provides a semi-quantitative description of the kinetics for our ideal model system. The photokinetics of our model system is representative of typical atmospheric aerosols; that is, of typical optical properties of these particles. For example, similar quantitative results are predicted for aqueous droplets (Supplementary Fig. 7). The acceleration of the kinetics we find in the visible range is predicted to be even more pronounced in the UV range of the solar spectrum (Supplementary Fig. 7). Many aerosol particles are non-spherical. However, for particles with different shapes but the same volumes one finds quantitatively similar nanofocusing effects as for droplets. Nanofocusing also affects surface reactions since the strong intensity enhancement in forward direction shown for the internal field in Fig. $4 \mathrm{~b}$ extends to the external field near the surface (not shown). The ability to measure and thus quantify the kinetics of the light-induced step in photochemical reactions in aerosol particles is of fundamental importance for atmospheric chemistry, where chemical processes are largely driven by sunlight. The diverse and complex processes (for example, transport and surface phenomena) in atmospheric aerosol particles require direct measurement methods as the one introduced here because simple models are of limited applicability.

The introduction of single-droplet PA spectroscopy in the present study finally makes the direct observation of the photoactive step possible. Single-droplet PA was previously deemed not feasible because of sensitivity and background issues. Here we demonstrate the viability of this new method and its very high sensitivity $\left(C_{\mathrm{abs} \text { min }}=0.0037 \mu \mathrm{m}^{2}\right)$ enabling studies even of single nanodroplets $(10 \mathrm{al})$. PA spectroscopy provides a general absorption method that can be used in any frequency range. The combination with laser trapping lets us follow the evolution of individual droplets under controlled conditions over extended periods of time (up to several days). This versatility enables fundamental studies on many different droplet systems relevant to atmospheric and technical processes. The investigation of droplet photokinetics is just one example where this new broadly applicable single-droplet method can make an important contribution.

\section{Methods}

PA measurements with microphone. The PA cell is made of brass and consists of a longitudinal PA-resonator (length $40 \mathrm{~mm}$, diameter $4 \mathrm{~mm}$ ), which is connected to two buffer volumes with acoustical baffles for sound insulation (Fig. 1a) ${ }^{45}$. A sensitive microphone (EK 23029, Knowles) is used with a custom-made preamplifier. The output signal is recorded by a lock-in amplifier (Stanford, SR 830).

PA measurements with tuning fork. The distance between the two tines of the tuning fork (Q $32.768 \mathrm{kHz}$ TC 38 , AURIS) is $300 \mu \mathrm{m}$. Each tine has a width of $600 \mu \mathrm{m}$, a thickness of $340 \mu \mathrm{m}$ and a length of $3.8 \mathrm{~mm}$. The quartz tuning fork acts as the resonant acoustic transducer, which generates an electric signal on resonant excitation by an acoustic wave due to the piezoelectric effect ${ }^{46}$. The signal recording is identical to the microphone set-up except for the more precise reference frequency adapted to the higher $Q$-factor.

Aerosol generation and materials. To study photokinetics in single droplets, solutions of the photoactive dye VIS441 (Cyanine dye with formula $\mathrm{NaC}_{17} \mathrm{H}_{25} \mathrm{~N}_{3} \mathrm{O}_{5} \mathrm{~S}_{3}$ and molar mass 470, QCR solutions) in TEG solvent (ACROS organics, 99.5\%) are nebulized with a medical nebulizer (Pari, PARI Boy SX). A concentration of $4.55 \mathrm{gl}^{-1}$ VIS441 in TEG is used. For measurements on pure solvent droplets, pure TEG is nebulized. The Supplementary Fig. 4 shows an UV/VIS spectrum of a bulk solution of VIS441 in TEG and of pure TEG solvent, respectively.

Droplet sizing. The particle size is determined from excitation laser light scattered elastically by the droplet. The scattered light intensity is collected for scattering angles between $76.5^{\circ}$ and $103.5^{\circ}$ and focused onto a CMOS camera (Thorlabs, DCC1645C, $1280 \times 1024$ pixels) using a camera objective (Super Carenar, focal length $=50 \mathrm{~mm}, f$-number $=1.7)$. The particle size is retrieved by fitting calculated phase functions to experimental ones using Mie theory ${ }^{41,44}$. The sizing of sub-micron-sized droplets is difficult because only few fringes are left in the scattering pattern (for example, Fig. 1e). Larger particles exhibit brighter scattering images and many more fringes (Supplementary Fig. 3), which makes sizing easier. We estimate uncertainties in the droplet radius of about half the wavelength.

Calculation of droplet absorbance. The PA signal $S$ is assumed to be proportional to the power $P_{\text {abs }}$ absorbed by the droplet, which is located at the centre of a Gaussian excitation laser beam (beam waist radius of $87 \mu \mathrm{m}$ and cross-section $q_{\mathrm{L}}=11,889 \mu \mathrm{m}^{2}$ ) with incident power $P$

$$
S \propto P_{\mathrm{abs}}=I_{0} \cdot C_{\mathrm{abs}}=P \cdot \frac{C_{\mathrm{abs}}}{q_{\mathrm{L}}}
$$

$I_{0}$ is the intensity incident on the droplet and $C_{\mathrm{abs}}$ is the droplet's absorption cross-section. The equivalent absorbance $A$ due to absorption is given by

$$
A=\ln \left(\frac{P}{P-P_{\mathrm{abs}}}\right)=\ln \left(\frac{q_{\mathrm{L}}}{q_{\mathrm{L}}-C_{\mathrm{abs}}}\right) \approx \frac{C_{\mathrm{abs}}}{q_{\mathrm{L}}}
$$

For a single droplet in the PA cell, the equivalent absorption coefficient is given by,

$$
\alpha=\frac{C_{\mathrm{abs}}}{V_{\text {res }}}
$$

where $V_{\text {res }}=0.5 \mathrm{~cm}^{3}$ is the volume of the PA-resonator. The absorption cross-section of the droplet is calculated from the Mie theory ${ }^{44}$ with a refractive index of the surroundings equal to 1 :

$$
C_{\mathrm{abs}}=\frac{2 \pi}{\varepsilon \cdot \mu \cdot \omega^{2}}\left[\sum_{j}(2 j+1)\left(\operatorname{Re}\left\{a_{j}(x, m)+b_{j}(x, m)\right\}-\left|a_{j}(x, m)\right|^{2}-\left|b_{j}(x, m)\right|^{2}\right)\right]
$$

Here $a_{n}$ and $b_{n}$ are the scattering coefficients, $x=\frac{2 \pi a}{\lambda}$ is the size parameter, $a$ is the droplet radius, $\lambda$ its wavelength of light in vacuum, $\omega$ is the angular frequency of the light, $\varepsilon$ and $\mu$ are the permittivity and the permeability, respectively, of the droplet, and $m=n+i k$ is the droplet's complex index of refraction at the wavelengths of the excitation laser $(\lambda=445 \mathrm{~nm})$. The latter is determined from UV/VIS absorption and refractometric measurements of VIS441/TEG bulk solutions and a pure TEG solution using Kramers-Kronig inversion. The refractive index of the VIS441/TEG solution for a dye concentration $4.55 \mathrm{gl}^{-1}$ and the pure TEG solvent are $n+i k=1.463+i \cdot 0.0062$ and $n_{\mathrm{s}}+i k_{\mathrm{s}}=1.460+i \cdot 0.0000$, respectively. The refractive index of the VIS441/TEG solution (dye concentration $4.55 \mathrm{gl}^{-1}$ ) in the UV/VIS range is provided in the Supplementary Fig. 5. For other dye concentrations (see photokinetics), it is assumed to depend linearly on the dye concentration.

Calculation of droplet photokinetics. The droplet photokinetics is described by the following rate equation

$$
\frac{\mathrm{d} N}{\mathrm{~d} t}=-p \cdot \frac{I(\boldsymbol{r})}{h v} \cdot \sigma(\boldsymbol{r}) \cdot N(\boldsymbol{r})
$$

with the number density of reactant molecules $N$, Planck's constant $h$, excitation laser frequency $v$, molecular absorption cross-section $\sigma$ and the quantum yield 
$p$. Here $\boldsymbol{r}$ denotes the location within the droplet and $I$ is the local field intensity. Both $I$ and $\sigma$ depend on the complex index of refraction, which in turn depends on the number density $N$, so that the rate law is no longer pseudo first order. The power absorbed by the droplet is given by the rate of absorption integrated over the droplet's volume $V$

$$
P_{\text {abs }}(t)=I_{0} \cdot C_{\text {abs }}(N)=-h v \cdot \int p^{-1} \frac{\mathrm{d} N}{\mathrm{~d} t} \mathrm{~d} V
$$

Assuming fast diffusion, that is, $N \neq N(\boldsymbol{r})$, we obtain:

$$
\frac{\mathrm{d} N}{\mathrm{~d} t}=-f \cdot V^{-1} C_{\mathrm{abs}}(N)
$$

where $f=p \cdot I_{0} / h v$ is the product of incident photon flux and reaction probability. Equation (7) is integrated using a 4th order Runge-Kutta method with the time-dependent PA signal given by equation (1). The corresponding inverse first half-lives of the PA signal as a function of droplet radius are shown as a full blue line in Fig. 4a.

To illustrate the effect of nanofocusing, we compare the above model (full blue line in Fig. 4a) with a model that neglects the influence of nanofocusing (dashed red line in Fig. 4a). This model is obtained from equations (5) and (6)

$$
C_{\text {abs }}=N(t) \int \frac{I(r)}{I_{0}} \sigma(\boldsymbol{r}) \mathrm{d} V
$$

by inserting the small particle limit ${ }^{44}$ for $\sigma$

$$
\sigma V^{-1}=\frac{\pi}{\lambda} \operatorname{Im}\left\{\frac{-18}{m^{2}+2}\right\}
$$

and a Beer-Lambert expression for the intensity distribution within the particle

$$
I(\boldsymbol{r})=I_{0} \cdot \exp \left\{-\frac{4 \pi k}{\lambda} \ell(\boldsymbol{r})\right\}
$$

where $\ell(\boldsymbol{r})=r \cos \theta+\sqrt{a^{2}-(r \sin \theta)^{2}}$ is the absorption path length at distance $r$ from the centre of the particle and at polar angle $\theta$ relative to the incident beam direction.

Statistical analysis of experimental photolysis data. To account for the uncertainties both in the particle radii and in the decay half-lives of the experimental data set (Supplementary Fig. 6), we perform a two-step maximum likelihood analysis. First, the distribution of particle radii $D(a)=\sum g_{i}(a)$ is analysed assuming a normally distributed error for the size determination,

$$
g_{i}(a)=\frac{\exp \left\{-\left(a-a_{i}\right)^{2} / 2 \sigma_{a}^{2}\right\}}{\sigma_{a} \sqrt{2 \pi}}
$$

with a constant s.d. of $\sigma_{a}=220 \mathrm{~nm}$. The local extrema in $D$ at $a_{k}$ divide the size range into sections with a lower and an upper half for each cluster of data, which are combined into a single section for isolated data points. For each section, we finally obtain the most probable values for particle radius and the inverse half-life as weighted averages over the particles with weights given by,

$$
w_{i, k}=\sigma_{t, i}^{-2} \int_{a_{k-1}}^{a_{k}} g_{i}(a) \mathrm{d} a / \sum_{i} \sigma_{t, i}^{-2} \int_{a_{k-1}}^{a_{k}} g_{i}(a) \mathrm{d} a
$$

This implies normally distributed errors for the experimental inverse half-lives with s.d. $\sigma_{t, i}$ ranging from about $10 \%$ for the most accurate measurements to about $50 \%$ for measurements with $\frac{S}{N L}<<10$ (typically small particles). The error bars in Fig. $4 \mathrm{a}$ were obtained by s.e. propagation.

\section{References}

1. Nie, W. et al. Polluted dust promotes new particle formation and growth. Sci. Rep. 4, 1-6 (2014).

2. George, C., Ammann, M., D’Anna, B., Donaldson, D. J. \& Nizkorodov, S. A. Heterogeneous photochemistry in the atmosphere. Chem. Rev. 115, 4218-4258 (2015).

3. Liu, F., Beames, J. M., Petit, A. S., McCoy, A. B. \& Lester, M. I. Infrared-driven unimolecular reaction of $\mathrm{CH}_{3} \mathrm{CHOO}$ Criegee intermediates to $\mathrm{OH}$ radical products. Science 345, 1596-1598 (2014).

4. Reed Harris, A. E. et al. Photochemical kinetics of pyruvic acid in aqueous solution. J. Phys. Chem. A 118, 8505-8516 (2014).

5. Finlayson-Pitts, B. J. Chlorine chronicles. Nat. Chem. 5, 724 (2013).

6. Monge, M. E. et al. Alternative pathway for atmospheric particles growth. Proc. Natl Acad. Sci. USA 109, 6840-6844 (2012).

7. Jimenez, J. L. et al. Evolution of organic aerosols in the atmosphere. Science 326, 1525-1529 (2009).

8. Lack, D. A. et al. Relative humidity dependence of light absorption by mineral dust after long-range atmospheric transport from the Sahara. Geophys. Res. Lett. 36, L24805 (2009).

9. Laskin, A. et al. Reactions at interfaces as a source of sulfate formation in sea-salt particles. Science 301, 340-344 (2003).
10. Vaida, V., Kjaergaard, H. G., Hintze, P. E. \& Donaldson, D. J. Photolysis of sulfuric acid vapor by visible solar radiation. Science 299, 1566-1568 (2003).

11. Jacobson, M. Z. Strong radiative heating due to the mixing state of black carbon in atmospheric aerosols. Nature 409, 695-697 (2001).

12. Lelieveld, J. \& Crutzen, P. J. Influences of cloud photochemical processes on tropospheric ozone. Nature 343, 227-233 (1990).

13. Crutzen, P. J. \& Arnold, F. Nitric acid cloud formation in the cold Antarctic stratosphere: a major cause for the springtime 'ozone hole'. Nature 324, 651-655 (1986).

14. Tyndall, J. On the blue colour of the sky, and the polarization of light. Proc. $R$. Soc. 37, 384-394 (1869).

15. Benincasa, D. S., Barber, P. W., Zhang, J.-Z., Hsieh, W.-F. \& Chang, R. K. Spatial distribution of the internal and near-field intensities of large cylindrical and spherical scatterers. Appl. Opt. 26, 1348-1356 (1987).

16. Symes, R., Sayer, R. M. \& Reid, J. P. Cavity enhanced droplet spectroscopy: principles, perspectives and prospects. Phys. Chem. Chem. Phys. 6, 474-487 (2004).

17. Cappa, C. D., Wilson, K. R., Messer, B. M., Saykally, R. J. \& Cohen, R. C. Optical cavity resonances in water micro-droplets: implications for shortwave cloud forcing. Geophys. Res. Lett. 31, L10205 (2004).

18. Brem, B. T., Gonzalez, F. C. M., Meyers, S. R., Bond, T. C. \& Rood, M. J. Laboratory-measured optical properties of inorganic and organic aerosols at relative humidities up to 95\%. Aerosol. Sci. Technol. 46, 178-190 (2012).

19. Preston, T. C. \& Signorell, R. Vibron and phonon hybridization in dielectric nanostructures. Proc. Natl Acad. Sci. USA 108, 5532-5536 (2011).

20. Preston, T. C. \& Signorell, R. From plasmon spectra of metallic to vibron spectra of dielectric nanoparticles. Acc. Chem. Res. 45, 1501-1510 (2012).

21. Hickstein, D. D. et al. Mapping nanoscale absorption of femtosecond laser pulses using plasma explosion imaging. Am. Chem. Soc. Nano 8, 8810-8818 (2014).

22. Goldmann, M., Miguel-Sánchez, J., West, A. H. C., Yoder, B. L. \& Signorell, R. Electron mean free path from angle-dependent photoelectron spectroscopy of aerosol particles. J. Chem. Phys. 142, 224304 (2015).

23. Ruth Signorell \& Jonathan, P. Reid (eds) Fundamentals and Applications in Aerosol Spectroscopy (CRC Press, 2011).

24. Mayer, B. \& Madronich, S. Actinic flux and photolysis in water droplets: Mie calculations and geometrical optics limit. Atmos. Chem. Phys. 4, 2241-2250 (2004).

25. Ray, A. K. \& Bhanti, D. D. Effect of optical resonances on photochemical reactions in microdroplets. Appl. Opt. 36, 2663-2674 (1997).

26. Ruggaber, A. et al. Modelling of radiation quantities and photolysis frequencies in the aqueous phase in the troposphere. Atmos. Environ. 31, 3137-3150 (1997).

27. Bott, A. \& Zdunkowski, W. Electromagnetic energy within dielectric spheres. J. Opt. Soc. Am. A 4, 1361-1365 (1987).

28. Penconi, M. et al. The use of chemical actinometry for the evaluation of the light absorption efficiency in scattering photopolymerizable miniemulsions. Photochem. Photobiol. Sci. 14, 308-319 (2015).

29. Nissenson, P., Knox, C. J. H., Finlayson-Pitts, B. J., Phillips, L. F. \& Dabdub, D Enhanced photolysis in aerosols: evidence for important surface effects. Phys. Chem. Chem. Phys. 8, 4700-4710 (2006)

30. Mills, C. T., Rowland, G. A., Westergren, J. \& Phillips, L. F. Quantum yields of $\mathrm{CO}_{2}$ and $\mathrm{SO}_{2}$ formation from $193 \mathrm{~nm}$ photo-oxidation of $\mathrm{CO}$ in a sulfuric acid aerosol. J. Photochem. Photobiol. A 93, 83-87 (1996).

31. Kitagawa, F. \& Kitamura, N. A laser trapping-spectroscopy study on the photocyanation of perylene across a single micrometre-sized oil droplets/water interface: droplet-size effects on photoreaction quantum yield. Phys. Chem. Chem. Phys. 4, 4495-4503 (2002).

32. Barnes, M. D., Whitten, W. B. \& Ramsey, J. M. Enhanced fluorescence yields through cavity quantum-electrodynamic effects in microdroplets. J. Opt. Soc. Am. B 11, 1297-1304 (1994)

33. Taflin, D. C. \& Davis, E. J. A study of aerosol chemical reactions by optical resonance spectroscopy. J. Aerosol. Sci. 21, 73-86 (1990).

34. Ward, T. L., Zhang, S. H., Allen, T. \& Davis, E. J. Photochemical polymerization of acrylamide aerosol particles. J. Colloid Interface Sci. 118, 343-355 (1987).

35. Sanford, T. J., Murphy, D. M., Thomson, D. S. \& Fox, R. W. Albedo measurements and optical sizing of single aerosol particles. Aerosol. Sci. Technol. 42, 958-969 (2008).

36. Cotterell, M. I., Mason, B. J., Preston, T. C., Orr-Ewing, A. J. \& Reid, J. P. Optical extinction efficiency measurements on fine and accumulation mode aerosol using single particle cavity ring-down spectroscopy. Phys. Chem. Chem. Phys. 17, 15843-15856 (2015).

37. Haisch, C. Photoacoustic spectroscopy for analytical measurements. Meas. Sci. Technol. 23, 012001 (2012). 
38. Haisch, C., Menzenbach, P., Bladt, H. \& Niessner, R. A wide spectral range photoacoustic aerosol absorption spectrometer. Anal. Chem. 84, 8941-8945 (2012).

39. Lack, D. A. et al. Aircraft instrument for comprehensive characterization of aerosol optical properties, part 2: black and brown carbon absorption and absorption enhancement measured with photo acoustic spectroscopy. Aerosol. Sci. Technol. 46, 555-568 (2012).

40. Gyawali, M. et al. Photoacoustic optical properties at UV, VIS, and near IR wavelengths for laboratory generated and winter time ambient urban aerosols. Atmos. Chem. Phys. 12, 2587-2601 (2012).

41. David, G. et al. Stability of aerosol droplets in Bessel beam optical traps under constant and pulsed external forces. J. Chem. Phys. 142, 154506 (2015).

42. Thanopulos, I., Luckhaus, D., Preston, T. C. \& Signorell, R. Dynamics of submicron aerosol droplets in a robust optical trap formed by multiple Bessel beams. J. Appl. Phys. 115, 154304 (2014).

43. Li, T., Kheifets, S., Medellin, D. \& Raizen, M. G. Measurement of the instantaneous velocity of a brownian particle. Science 328, 1673-1675 (2010).

44. Bohren, C. F. \& Huffman, D. R. Absorption and Scattering of Light by Small Particles (John Wiley \& Sons, 1998).

45. Beck, H. A., Niessner, R. \& Haisch, C. Development and characterization of a mobile photoacoustic sensor for on-line soot emission monitoring in diesel exhaust gas. Anal. Bioanal. Chem. 375, 1136-1143 (2003).

46. Kosterev, A. A., Tittel, F. K., Serebryakov, D. V., Malinovsky, A. L. \& Morozov, I. V. Applications of quartz tuning forks in spectroscopic gas sensing. Rev. Sci. Instrum. 76, 043105 (2005).

47. Raspet, R., Slaton, W. V., Arnott, W. P. \& Moosmüller, H. Evaporationcondensation effects on resonant photoacoustics of volatile aerosols. J. Atmos. Oceanic Technol. 20, 685-695 (2003).

\section{Acknowledgements}

This work was supported by the Swiss National Science Foundation (SNSF grant nr. 200020_159205), ETH Zurich and the TUM International Graduate School of
Science and Engineering (IGSSE). We are very grateful to Dr David Luckhaus for support in the data analysis and to Prof. Markus Sigrist for helpful discussions concerning the PA set-up. We would like to thank Guido Grassi and Daniel Zindel from the analytical service at ETH and the electronic and mechanical workshops at ETH and TUM for their technical support.

\section{Author contributions}

J.W.C. implemented the experimental set-up and performed the measurements. K.M.T. and C.H. designed the PA cell and contributed to the experimental set-up and initial test measurements. J.W.C. and R.S. analysed the data. R.S. conceived the project, performed the calculations and wrote the manuscript.

\section{Additional information}

Supplementary Information accompanies this paper at http://www.nature.com/ naturecommunications

Competing financial interests: The authors declare no competing financial interests.

Reprints and permission information is available online at http://npg.nature.com/ reprintsandpermissions/

How to cite this article: Cremer, J. W. et al. Photoacoustics of single laser-trapped nanodroplets for the direct observation of nanofocusing in aerosol photokinetics. Nat. Commun. 7:10941 doi: 10.1038/ncomms10941 (2016).

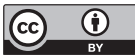

This work is licensed under a Creative Commons Attribution 4.0 International License. The images or other third party material in this article are included in the article's Creative Commons license, unless indicated otherwise in the credit line; if the material is not included under the Creative Commons license, users will need to obtain permission from the license holder to reproduce the material. To view a copy of this license, visit http://creativecommons.org/licenses/by/4.0/ 